\title{
Physics at an Upgraded Proton Driver at Fermilab
}

\author{
S. Geer, Fermilab
}

\section{Introduction}

The accelerator-based particle physics program in the US is entering a period of transition. This is particularly true at Fermilab which for more than two decades has been the home of the Tevatron Proton-Antiproton Collider, the World's highest energy hadron collider. In a few years time the energy frontier will move to the LHC at CERN. Hence, if an accelerator-based program is to survive at Fermilab, it must evolve. Fermilab is fortunate in that, in addition to hosting the Tevatron Collider, the laboratory also hosts the US accelerator-based neutrino program. The recent discovery that neutrino flavors oscillate has opened a new exciting world for us to explore, and has created an opportunity for the Fermilab accelerator complex to continue to address the cutting-edge questions of particle physics beyond the Tevatron Collider era.

The presently foreseen neutrino oscillation experiments at Fermilab (MiniBooNE [1] and MINOS [2]) will enable the laboratory to begin contributing to the Global oscillation physics program in the near future, and will help us better understand the basic parameters describing the oscillations. However, this is only a first step. To be able to pin down all of the oscillation parameters, and hopefully make new discoveries along the way, we will need high statistics experiments, which will require a very intense neutrino beam, and one or more very massive detectors. In particular we will require new MW-scale primary proton beams and perhaps ultimately a Neutrino Factory [3].

Plans to upgrade the Fermilab Proton Driver are presently being developed [4]. The upgrade project would replace the Fermilab Booster with a new 8 $\mathrm{GeV}$ accelerator with $0.5-2 \mathrm{MW}$ beam power, a factor of 15-60 more than the current Booster. It would also make the modifications needed to the Fermilab Main Injector (MI) to upgrade it to simultaneously provide $120 \mathrm{GeV}$ beams of $2 \mathrm{MW}$. This would enable a factor of 5-10 increase in neutrino beam intensities at the MI, whilst also supporting a vigorous $8 \mathrm{GeV}$ fixed-target program. In addition, a Proton Driver might also serve as a stepping-stone to future accelerators, both as an R\&D test bed and as an injector, with connections to the Linear Collider, Neutrino Factories, and a VLHC. Hence, although neutrino physics would provide the main thrust for the science program at an upgraded Fermilab proton source, the new facility would also offer exciting opportunities for other fixed-target particle physics (kaons, muons, neutrons, antiprotons, etc.) and a path towards new accelerators in the future.

\section{Neutrino Physics}

We now know that neutrino flavors oscillate and hence that neutrinos have nonzero masses and mixings. The Standard Model cannot accommodate neutrino mass terms, which require either the existence of right-handed neutrinos to create Dirac mass terms, or a violation of lepton number conservation to create Majorana mass terms (possible if neutrinos are their own antiparticle), or both. The observation that neutrino masses and mass splittings are tiny compared to the masses of any of the other fundamental fermions suggests radically new physics, which perhaps originates at the GUT or Planck Scale, or indicates the existence of new spatial dimensions beyond the three we know of. Whatever the origin of the observed neutrino masses and mixings is, it will certainly require a profound extension to our picture of the physical world. The first step in understanding this revolutionary new physics is to pin down the measurable parameters, and address the first round of basic questions:

Are there only three neutrino flavors, or do light sterile neutrinos exist? Are there any other deviations to three-flavor mixing?

There is one angle $\theta_{13}$ in the mixing matrix which is unmeasured. Is it non-zero?

We don't know the mass-ordering of the neutrino mass eigenstates. There are two possibilities, the so-called "normal" or "inverted" hierarchies. Which is right?

There is one complex phase $\delta$ in the mixing matrix which is accessible to neutrino oscillation measurements. If both $\theta_{13}$ and $\sin \delta$ are nonzero there will be CP Violation in the lepton sector. Is $\sin \delta$ non-zero ?

What precisely is the value of the lightest neutrino mass and are neutrino masses generated by Majorana mass terms, Dirac mass terms, or both? 
With the exception of the last question, all of these questions can in principle be addressed by accelerator-based neutrino oscillation experiments. The answers to these questions will guide our understanding of what lies beyond the Standard Model, and whether the new physics provides an explanation for the baryon asymmetry of the Universe (via leptogenesis), or provides deep insight into the connection between quark and lepton properties (via Grand Unified Theories), or perhaps leads to an understanding of one of the most profound questions in physics: Why are there three generations of quarks and leptons? In addition, the answers may well further challenge our picture of the physical world, and will certainly have important implications for our understanding of cosmology and the evolution of the early Universe.

\section{Present Fermilab Neutrino Physics Program}

There are five currently approved Fermilab experiments that will contribute to the Gobal neutrino oscillation program:

- MiniBooNE [1] is presently taking data at the Fermilab Booster. In the near future this experiment is expected to either confirm or refute the neutrino oscillation interpretation of the LSND result.

- MINOS [2] will start taking data early next year using a $0.25 \mathrm{MW}$ primary proton beam from the Fermilab MI. This experiment is expected to observe the "wiggle" in the oscillation spectra at the atmospheric-neutrino scale, and precisely determine the associated $\Delta \mathrm{m}^{2}$. MINOS is also expected to improve the sensitivity to a finite $\theta_{13}$ by a modest amount.

- MINERVA [5] has just received so-called "stage 1" approval at Fermilab. This experiment will measure neutrino interactions in the NuMI near hall, presumably in a few years from now.

- MUCOOL [6] is an ongoing accelerator R\&D experimental program to develop a muon ionization cooling channel needed for a Neutrino Factory.

- MIPP [7] is presently taking data at the MI. MIPP is a particle production experiment partly motivated by the need improved experimental input used by neutrino beam production simulations.
Thus, Fermilab has a very broad base on which to build a longer-term neutrino physics program.

\section{Future Fermilab Neutrino Physics Program}

An upgraded proton source enabling a $2 \mathrm{MW}$ beam to be extracted from the MI would facilitate a staged approach to long-baseline neutrino experiments at Fermilab. One possible sequence of steps (see Table 1) is:

- Step 0: The present long-baseline program (MINOS).

- Step 1: A 2 MW Proton Driver and a new very massive (off-axis?) experiment that probes $\sin ^{2} 2 \theta_{13}$ down to $0.001-0.002$, and if the result is positive, perhaps determines the mass hierarchy, and begins the search for CP Violation.

- Step 3: A second generation Superbeam experiment optimized to complete the physics program opened up by the first generation experiment $\underline{\mathrm{OR}}$ a Neutrino Factory, as required. The Neutrino Factory would enable the first observation of transitions between electron-neutrinos and muonneutrinos in an appearance experiment even if $\sin ^{2} 2 \theta_{13}=0$, and if $\sin ^{2} 2 \theta_{13}>0\left(10^{-4}\right)$ would enable its value to be measured, the mass hierarchy to be determined, and a sensitive search for maximal CP violation to be conducted.

Table 1: Possible evolution of the Global longbaseline neutrino oscillation program.

\begin{tabular}{|l|l|l|}
\hline Experiments & $\begin{array}{l}\text { Beam } \\
\text { Power } \\
\text { (MW) }\end{array}$ & $\begin{array}{l}\sin ^{2} 2 \theta_{13} \\
\text { Sensitivity } \\
(90 \% \text { CL) }\end{array}$ \\
\hline Near-Term & 0.15 & 0.04 \\
\hline OPERA [8] & 0.15 & 0.03 \\
\hline ICARUS [8] & $0.25 \rightarrow 0.4$ & 0.05 \\
\hline MINOS [9] & & \\
\hline Medium-Term & 0.15 & 0.02 \\
\hline CNGS [10] & 0.8 & 0.006 \\
\hline T2K & 0.4 & 0.004 \\
\hline NOvA [11] & & \\
\hline Medium-Term + & 4 & $\sim 0.001$ \\
\hline T2HK & 2 & $\sim 0.001$ \\
\hline Super-NOvA & 4 & 0.0016 \\
\hline CERN SPL & 0.04 & $\sim 0.001$ \\
\hline Beta Beam & & \\
\hline Long-Term & 4 & $<0.0001$ \\
\hline Neutrino Factory & 4 & \\
\hline
\end{tabular}


In addition to a staged long-baseline neutrino oscillation physics program, an upgraded proton source would also facilitate a continued and expanded short baseline program. The present Booster-based program at Fermilab is limited by proton economics, and this limitation will get worse once the NuMI program begins next year. An upgraded proton source would ease the constraints, and provide flexibility both to exploit surprises (if MiniBooNE confirms LSND for example) and support a broader range of smaller experiments (e.g. cross-section measurements, searches for magnetic moments or exotic neutrino interactions). As an example, in Table 2 neutrino beam intensities and event rates for a Decay at Rest source based on a Fermilab Proton Driver are shown to be a factor of a few higher than those at a corresponding hypothetical source at the soon to be completed SNS facility at Oak Ridge.

Table 2: Comparison of Decay at Rest (DAR) neutrino sources based on the new SNS facility under construction at Oak Ridge, and the upgraded 8 $\mathrm{GeV}$ Proton Driver under discussion at Fermilab. Table from [12].

\begin{tabular}{|l|l|l|}
\hline Parameters & $\begin{array}{l}\text { FNAL 2MW } \\
\text { at } 8 \mathrm{GeV}\end{array}$ & $\begin{array}{l}\text { SNS1.4 MW } \\
\text { at } 1.3 \mathrm{GeV}\end{array}$ \\
\hline $\mathrm{p} / \mathrm{yr}$ & $1.6 \times 10^{22}$ & $6.7 \times 10^{22}$ \\
\hline DAR $v / \mathrm{p}$ & 1.5 & 0.13 \\
\hline DAR $v / \mathrm{yr}$ & $2.3 \times 10^{22}$ & $0.9 \times 10^{22}$ \\
\hline$v_{\mathrm{e}}($ events/yr) & 8900 & 3500 \\
\hline anti- $v_{\mu}($ evnts/yr) & 1500 & 600 \\
\hline$v_{\mu}($ events/yr) & 3200 & 1200 \\
\hline
\end{tabular}

In summary, the neutrino program that would be supported by an upgraded 2 MW proton source at Fermilab is likely to consist of a multi-phase program with at least a handful of experiments that would provide World-class cutting-edge physics for at least a couple of decades or longer.

\section{A Broader Proton Driver Program}

Although neutrino physics provides a compelling case for a Proton Driver upgrade at Fermilab, diversity is also important. With an intensity-frontier facility there are other potentially big discovery type experiments and important measurements that could also be supported. A previous Fermilab Proton Driver physics study [13] has identified some of these opportunities. Some examples are:
- Lepton Flavor Violation Searches. For example: $\mu \rightarrow$ e $\gamma, \mu \rightarrow$ e $\gamma \gamma, \mu \rightarrow$ e conversion. These rare muon processes provide important constraints on physics beyond the Standard Model that complement direct searches at the energy frontier.

- Precision measurements of the CKM Matrix. For example: comparison of B-physics and rare kaon decay measurements, and the search for CP Violation in the hyperon system. Grand Unified Theories provide a connection between mixing in the quark and lepton sectors. Hence, as we learn more about neutrino mixing it is likely that the importance of detailed CKM measurements will be increased since, together with the neutrino mixing measurements, they provide a test of GUT models.

- Interface between particle and nuclear physics. For example: Flavor-dependent and polarized nucleon structure functions, the search for exotic hadrons, nuclear shadowing measurements, and the pion structure function. In addition to being of interest in their own right, many of these measurements are also important to reduce systematic uncertainties in the analysis of purely particle physics measurements.

These are just a few examples from a much longer list which includes topics as diverse as AcceleratorBased Particle-Astrophysics and R\&D in Medical Physics. Over time the proton accelerator complex at Fermilab could be further upgraded to include facilities that provide intense secondary beams (muons, antiprotons, neutrons, etc) and hence support a broad community.

\section{Summary}

An upgraded Fermilab proton source, consisting of a 2 MW Proton Driver and upgrades to the MI, is currently under study. The new source would support a World-class cutting edge neutrino program for decades to come, together with a broader program at the intensity frontier.

\section{References}

[1] http://www-boone.fnal.gov/

[2] http://www-

numi.fnal.gov/public/minosexperiment.html

[3] S. Geer, Phys. Rev. D57, 6989 (1998) M.M. Alsharo'a et al., Phys. Rev. ST Accel. Bemas 6, 081001 (2003).

[4] R. Kephart, contribution to these proceedings.

[5] http://www.pas.rochester.edu/ ksmcf/minerva/ 
[6]http://www.fnal.gov/projects/muon_collider/cool/ cool.html

[7] http://ppd.fnal.gov/experiments/e907/e907.htm

[8] Kamatsu, Miggliozzi, Terranova., J.Phys G29

443, 2003.

[9] Diwan, Messier, Viran, Wai., NUMI-L-714

[10] Rubbia, Sala., hep-ph/0207084

[11] http://www-off-axis.fnal.gov/

[12] Brice, Geer, Paul, Tayloe., in preparation.

[13] C. Ankenbrandt et al., "Physics Study Group

Report on Physics Potential at FNAL with Stronger

Proton Sources", June 27, 2001. 